\title{
DESEMPENHO DE MÉTODOS E PROCESSOS DE AMOSTRAGEM PARA AVALIAÇÃO DE DIVERSIDADE EM FLORESTA OMBRÓFILA MISTA
}

\author{
Ana Paula Dalla Corte ${ }^{1}$, Carlos Roberto Sanquetta ${ }^{2}$, Afonso Figueiredo Filho ${ }^{3}$, \\ Thaiza Karine Pereira ${ }^{4}$, Alexandre Behling ${ }^{5}$ \\ ${ }^{1}$ Eng $^{\mathrm{a}}$. Florestal, Dra ${ }^{\mathrm{a}}$, Depto. de Ciências Florestais, UFPR, Curitiba, PR, Brasil - anacorte@ufpr.br \\ ${ }^{2}$ Eng. Florestal, Dr., Depto. de Ciências Florestais, UFPR, Curitiba, PR, Brasil - sanquetta@ufpr.br \\ ${ }^{3}$ Eng. Florestal, Dr., Depto. de Eng. Florestal, Universidade Estadual do Centro-Oeste, Irati, PR, Brasil - afig @ufpr.br \\ ${ }^{4}$ Acadêmica de Eng. Florestal, UFPR, Curitiba, PR, Brasil - thaizak.p@gmail.com \\ ${ }^{5}$ Eng. Florestal, Mestrando em Eng. Florestal, UFPR, Curitiba, PR, Brasil - alexandre.behling@ yahoo.com.br
}

Recebido para publicação: 18/01/2013 - Aceito para publicação: 06/09/2013

\begin{abstract}
Resumo
Este trabalho objetivou testar tamanhos, processos de distribuição e intensidades de amostragem, com a finalidade de indicar os melhores tratamentos para aplicação em levantamentos que visam expressar a diversidade florestal. Foram utilizados dados da FLONA de Irati, Paraná, e ao todo foram analisados 28 tratamentos. Os resultados foram analisados considerando o número de espécies, o número de indivíduos amostrados e os índices de Margalef, Shannon e de Simpson. A curva do coletor (ou curva espéciesárea) foi construída sob três abordagens: ordem numérica sequencial qualquer das parcelas; ordem crescente do número de espécies em cada parcela; e ordem da parcela com maior número de espécies para a de menor número amostradas. O processo aleatório representou melhor o número de espécies em áreas amostradas menores, ao passo que o sistemático em áreas amostradas maiores. Os índices de Simpson e de Shannon não tiveram sensibilidade para representar as variações encontradas em relação ao censo. $\mathrm{O}$ índice de Margalef apresentou sensibilidade melhor para expressar a diversidade de espécies presentes na área. A curva do coletor mostrou-se facilmente manipulável para representar uma situação favorável ou desfavorável para a análise da suficiência amostral.

Palavras-chave: Curva do coletor; Simpson; Shannon; Margalef.
\end{abstract}

\begin{abstract}
Performance of methods and sampling procedures in order to evaluate forest diversity in Araucaria forest. This research aimed at testing different plot and sample sizes, as well the sampling design to evaluate diversity indexes in a natural forest. The study was carried out in the Irati National Forest, Paraná. A combination of sampling schemes resulted in 28 treatments, which were evaluated considering the number of species, the number of sampled individuals and calculation of the Margalef, Shannon and Simpson indexes. The species accumulation curve was carried out based on three approaches: random sequential numerical order, ascending order of species number in each plot, and order of the parcel with the highest number of species for the smaller number of species. The random sample design represented at best the number of species in smaller areas (richness), and the systematic sample design represented better the biggest sampled areas. The Simpson index and Shannon index were not sensitive to represent the variations found in relation to the census. The Margalef index had better sensitivity to express species diversity of the forested area. The species accumulation curve proved its feasibility to represent a favorable and sufficient sampling size.
\end{abstract}

Keywords: Collector curve; Simpson; Shannon; Margalef.

\section{INTRODUÇÃO}

Vários foram os estudos com o enfoque de observar tamanhos e formas de unidades amostrais para inventários em florestas nativas. Dentre esses, devem-se destacar Silva (1980), em uma floresta tropical na região do Baixo Tapajós, no estado do Pará; Mello (1995), em um remanescente de floresta nativa no município de Lavras, Minas Gerais; Bonetes (2003), na Floresta Nacional de Chapecó, no 
município de Guatambu, Santa Catarina; Ubialli et al. (2009), em uma Floresta Ecotonal na região norte mato-grossense; e Oda-Souza et al. (2010), que avaliaram a influência do tamanho e forma da unidade amostral sobre a estrutura de dependência espacial em quatro formações florestais do estado de São Paulo, entre outros.

Ademais, medir $100 \%$ da população, muitas vezes, torna-se inviável sob o ponto de vista de tempo de execução e, consequentemente, dos recursos dispendidos para tanto. Destarte, a grande maioria dos trabalhos com esse enfoque acaba recorrendo às técnicas de amostragem. Nesse contexto, Sanquetta et al. (2009) expõem que o sucesso na obtenção de informações precisas oriundas de um inventário florestal está ligado à definição de processos e métodos de amostragem, bem como à intensidade amostral adequada.

Ainda, vários são os autores que têm levantado a dúvida sobre a confiabilidade de informações geradas em trabalhos fitossociológicos. Moreira (2007) e Cielo Filho e Santin (2002) destacam que as tabelas de fitossociologia apresentadas comumente em trabalhos de levantamentos fitossociológicos, em geral, apresentam pouca confiabilidade para traduzir os valores absolutos e também relativos das espécies, uma vez que eles podem ser modificados de forma considerável para uma mesma área, apenas com redistribuição das parcelas, ou com a alteração do seu formato ou tamanho. Nesse sentido, Durigan (2009) destaca que os estudos de diversidade e suas comparações exigem um esforço amostral alto, sendo que esse esforço amostral impactará significativamente os resultados produzidos.

Sabe-se que o tamanho das parcelas é um fator chave na realização de um inventário florestal. Singh (1974), ao estudar os efeitos que as dimensões e formas das unidades amostrais causam sobre a precisão das estimativas, concluiu que, nas florestas tropicais, tanto a dimensão como a forma exercem influência importante sobre o coeficiente de variação. Morais Filho et al. (2003) citaram que, de modo geral, os coeficientes de variação decrescem como função inversa do tamanho da parcela, e em consequência o número de parcelas necessárias para o mesmo grau de precisão é mais elevado quanto menor a parcela.

Scolforo (2006) orienta que, em florestas nativas que têm grande variabilidade, deve-se evitar o uso de parcelas pequenas, já que pequenas unidades podem resultar em grande numero de parcelas zero e, nesse caso, a teoria da distribuição normal torna-se inadequada. Já Durigan (2009) defende que o desenho amostral para a caracterização de comunidades vegetais deve ser estabelecido em função do objetivo da amostragem e da variável escolhida, de modo a fielmente representar a comunidade a ser estudada. Sanquetta et al. (2009) complementaram que o processo de amostragem pode ser aleatório, estratificado ou sistemático, entre outros.

Diante do exposto, este trabalho foi desenvolvido com o objetivo de testar diferentes tamanhos de unidades amostrais, seus processos de distribuição e intensidades de amostragem, com a finalidade de indicar as melhores combinações para a representação da riqueza de espécies e diversidade, bem como testar o desempenho da curva do coletor sob diferentes tratamentos, a fim de melhor retratar a situação de um fragmento de Floresta Ombrófila Mista, na Flona de Irati, tendo como base o censo realizado na área em questão.

\section{MATERIAIS E MÉTODOS}

O estudo foi realizado no município de Fernandes Pinheiro, estado do Paraná, especificamente na Floresta Nacional (FLONA) de Irati (Figura 1). De acordo com Disperati (1986), a extensão territorial da FLONA de Irati é de 3.495 hectares, sendo 57,6\% ocupados por florestas com predominância de araucária e 37,5\% por plantios estabelecidos entre as décadas de 1940 e 1960 com Pinus elliottii Engelm (658,04 ha), Araucaria angustifolia (Bertol.) Kuntze (417,94 ha) e Pinus taeda L. (96,68 ha), dentre outras.

Segundo Mazza et al. (2005), a FLONA de Irati está situada no Segundo Planalto Paranaense, na zona fisiográfica de Irati, no paralelo $25^{\circ} 27^{\prime} 56^{\prime \prime}$ de latitude sul, com interseção com o meridiano $50^{\circ} 37$ '51" de longitude oeste. A altitude média é de $820 \mathrm{~m}$ acima do nível do mar, com relevo suavemente ondulado. O clima da região, segundo a classificação climática de Köppen, é do tipo Cfb Subtropical Úmido Mesotérmico -, caracterizado por verões amenos, presença frequente de geadas e ausência de estação seca (MAZZA et al., 2005).

A vegetação da área faz parte da unidade fitogeográfica Floresta Ombrófila Mista, caracterizada pela presença da espécie Araucaria angustifolia (Bertol.) Kuntze, além de Ocotea porosa (Nees \& Mart.) 
Barroso, Ilex paraguariensis A. St.-Hil., Mimosa scabrella Benth., Cedrella fissilis Vell. e Roupala brasiliensis Klotzsch, entre outras.

Dentro da área da FLONA, em 2002, foram instaladas parcelas permanentes com área amostrada de 25 hectares (Figura 1), visando estudar as mudanças do crescimento, da florística e da fitossociologia, com o auxílio do laboratório de Manejo Florestal do curso de Engenharia Florestal da Universidade Estadual do Centro-Oeste (UNICENTRO), Irati, Paraná.
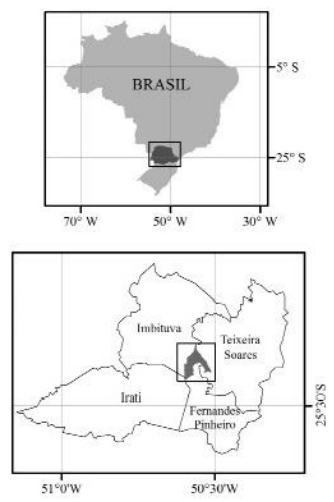
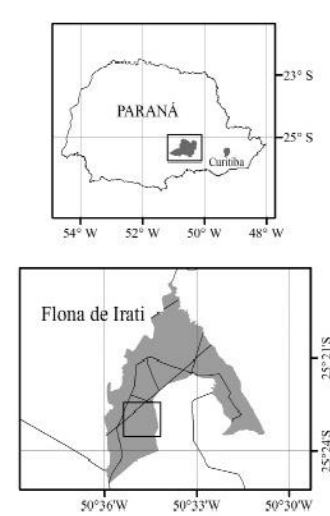

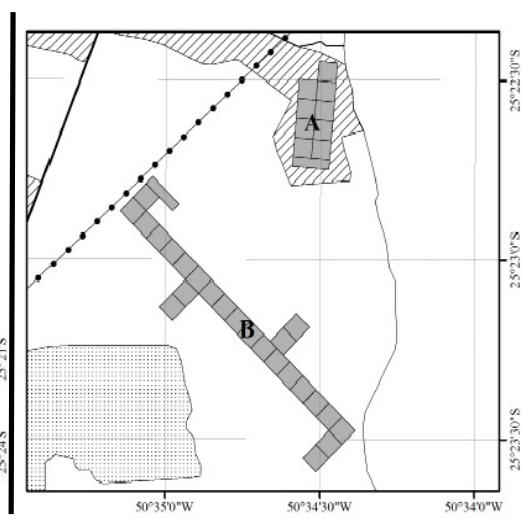

Figura 1. Localização da área de estudo na FLONA de Irati, estado do Paraná.

Figure 1. Location of the focused area at the Irati National Forest (FLONA), in Paraná State. Fonte: Rode (2008).

As parcelas foram implantadas com teodolito, sendo compostas por 25 blocos de 1 ha $(100 \times 100 \mathrm{~m})$. Cada bloco foi dividido em 4 parcelas de 0,25 ha $(50 \times 50 \mathrm{~m})$ e, para auxiliar a numeração e o mapeamento das árvores, cada parcela foi subdividida em 5 faixas de controle de 10 x $50 \mathrm{~m}$ (0,05 ha). Além disso, piquetes de concreto foram colocados a cada $50 \mathrm{~m}$ e piquetes de madeira a cada $10 \mathrm{~m}$.

Nessa área, as árvores com diâmetro à altura do peito (DAP) maior ou igual a $10 \mathrm{~cm}$ foram medidas em 2002 com fita métrica e posicionadas espacialmente em um sistema cartesiano X, Y, sendo remedidas em 2005 e 2008. Nas remedições, as novas árvores advindas da regeneração natural e que atingiram o limite de inclusão foram incorporadas aos dados já existentes, enquanto as árvores que morreram foram assinaladas (SAWCZUK, 2009). A remedição de 2008 constituiu-se no tratamento com abordagem de $100 \%$ dos indivíduos arbóreos, utilizada como parâmetro da população.

\section{Simulações de tratamentos de amostragem}

Foram considerados diferentes tratamentos de avaliação, conforme descritos a seguir, por meio dos fatores de variação.

Fator 1 - Área amostrada

Visando padronizar as combinações realizadas, foram definidas duas áreas totais amostradas para as comparações. Na primeira, foi fixada uma área de 2 ha medidos, ou seja, $8 \%$ da população. $\mathrm{Na}$ segunda, fixou-se a área de 4 ha medidos, ou seja, 16\%. Dessa forma, o número de parcelas foi calculado com base em sua dimensão e na área que deveria ser amostrada em cada um dos tratamentos descritos. Essa escolha foi baseada no trabalho de Magurran (2011), o qual afirma que, usualmente, a melhor abordagem é padronizar o tamanho das amostras.

Fator 2 - Tamanho das parcelas

Foram consideradas parcelas com 7 dimensões distintas, sendo elas apresentadas na tabela 1. A cada dimensão de parcela foi atribuído um tratamento, entre 2 a 8 , sendo aplicado para a intensidade de amostragem de 2 ha e 4 ha, gerando, assim, 14 combinações testadas. 
Tabela 1. Dimensões das parcelas, tamanhos de parcelas e quantidades.

Table 1. Plot dimensions, plot size and sample size.

\begin{tabular}{ccccc}
\hline Tratamento & $\begin{array}{c}\text { Tamanho da } \\
\text { parcela }\end{array}$ & $\begin{array}{c}\text { Área da parcela } \\
\left(\mathbf{m}^{2}\right)\end{array}$ & $\begin{array}{c}\text { Número de } \\
\text { parcelas - 2ha }\end{array}$ & $\begin{array}{c}\text { Número de } \\
\text { parcelas - 4ha }\end{array}$ \\
\hline T1 & \multicolumn{2}{c}{ Censo florestal } \\
T2 & $10 \times 50 \mathrm{~m}$ & 500 & 40 & 80 \\
T3 & $10 \times 100 \mathrm{~m}$ & 1.000 & 20 & 40 \\
T4 & $20 \times 20 \mathrm{~m}$ & 400 & 50 & 100 \\
T5 & $20 \times 25 \mathrm{~m}$ & 500 & 40 & 80 \\
T6 & $20 \times 50 \mathrm{~m}$ & 1.000 & 20 & 40 \\
T7 & $30 \times 35 \mathrm{~m}$ & 1.050 & 19 & 38 \\
T8 & $25 \times 40 \mathrm{~m}$ & 1.000 & 20 & 40 \\
\hline
\end{tabular}

Fator 3 - Processo de amostragem

Testou-se o desempenho dos processos de amostragem aleatório e sistemático. Dessa maneira, as 14 combinações mencionadas foram testadas para esses dois processos, tendo-se, finalmente, 28 tratamentos analisados.

A distribuição aleatória e sistemática das parcelas foi estabelecida com o software ArcMap 9.3® (ESRI, 2006), tendo como base o mapa de limite da área dos 25 hectares. A figura 2 apresenta o tratamento 8 como exemplo das combinações realizadas. As figuras $2 \mathrm{a}$ e $2 \mathrm{~b}$ mostram 20 parcelas com dimensão de $25 \times 40 \mathrm{~m}$, totalizando 2 ha de área inventariada, distribuídas de forma aleatória (a) e sistemática (b) sobre a área do censo. As figuras $2 \mathrm{c}$ e $2 \mathrm{~d}$ apresentam 40 parcelas com dimensão de 25 x $40 \mathrm{~m}$, totalizando 2 ha de área inventariada, distribuídas de forma aleatória (c) e sistemática (d) sobre a área do censo.

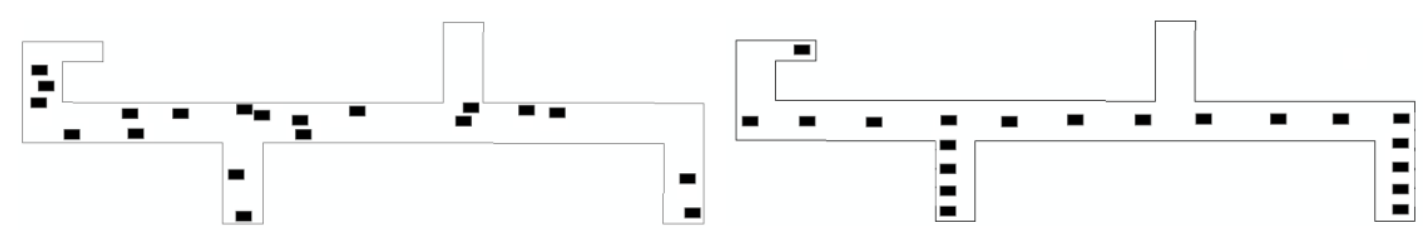

(a) 2 hectares, processo aleatório, 20 parcelas de 25 x 40 metros. (b) 2 hectares, processo sistemático, 20 parcelas de $25 \times 40$ metros.

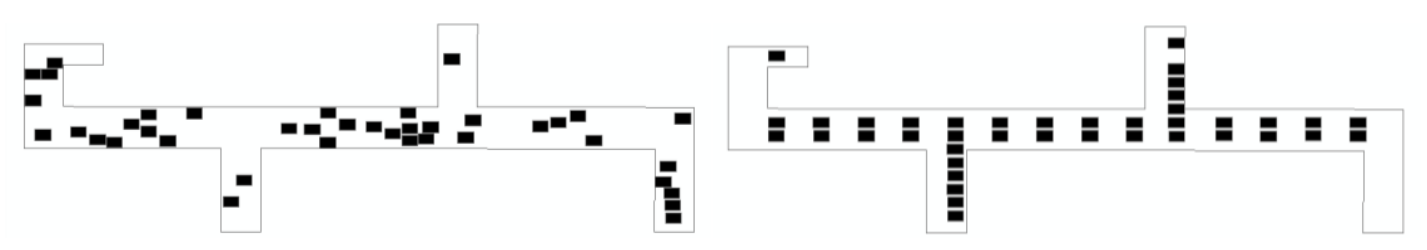

(c) 4 hectares, processo aleatório, 40 parcelas de 25 x 40 metros.

(d) 4 hectares, processo sistemático, 40 parcelas de $25 \times 40$ metros.

Figura 2. Exemplo das combinações aplicadas para avaliação do tratamento 8.

Figure 2. Example of combinations applied to evaluate the treatment 8.

Em seguida, como cada individuo foi posicionado em coordenadas $\mathrm{X}$ e $\mathrm{Y}$ na área do censo (25 ha), realizou-se uma interseção entre as áreas das parcelas e os indivíduos do censo, visando simular a realização do inventário florestal em cada um dos 28 tratamentos avaliados. Dessa forma, foi identificada a composição florística da testemunha (censo) e dos tratamentos, expressas por nomes populares e científicos, famílias e gêneros e variáveis dendrométricas. Os dados foram exportados para uma planilha Excel, perfazendo um tratamento de avaliação. 
O processamento desses dados foi realizado com o software FlorExel (OPTIMBER, 2013). Nesse processamento, foram computados: número de indivíduos amostrados, número de espécies e os índices de Margalef, Shannon e de Simpson.

O Índice de Margalef $\left(\mathrm{R}_{1}\right)$ é um índice alfa que trata do número total de indivíduos e da riqueza específica. Estima a biodiversidade baseado na distribuição numérica dos indivíduos de cada espécie diferente. Quanto maior o valor do índice, maior é a biodiversidade da área (KANIESKI, 2010).

O Î́ndice de Shannon, ou Shannon-Wiener ou ainda Shannon-Weaver (H'), é um índice alfa que mostra a diversidade de espécies. A vantagem é que esse índice leva em consideração o número das espécies e sua equitabilidade, sendo afetado por espécies raras (SANTOS, 2009). Esse índice varia entre 1,5 e 3,5, sendo pouco comum a ultrapassagem de 4,5 (GUAPYASSÚ, 1994).

O Índice de Simpson (D) é um índice alfa que representa a probabilidade de dois indivíduos escolhidos aleatoriamente de uma amostragem pertencerem a uma mesma espécie, mostrando um grau de dominância, significando que quanto maior o valor do índice, maior a dominância por uma ou poucas espécies (KANIESKI, 2010). Normalmente é expresso como 1 - D ou 1/D, situando-se entre 0 e 1, em que quanto mais próximo de 1, maior a diversidade da comunidade estudada (GUAPYASSÚ, 1994).

\section{Construção da Curva do Coletor ou Curva Espécies-Área}

Foram construídas as curvas do coletor para cada um dos 28 tratamentos avaliados. Para cada tratamento, foram consideradas três abordagens distintas para a construção do gráfico. Esse teste baseouse nas afirmações de Colwell e Coddington (1994) e Martins e Santos (1999) de que a arbitrariedade da ordem de entrada das unidades amostrais na construção da curva do coletor possibilita gerar uma curva diferente para cada ordenação. Adicionalmente, esses autores apresentam que qualquer interpretação a respeito da forma da curva, ou de variações nessa forma a partir de um padrão básico esperado, é inválida se essa curva tiver sido gerada a partir de uma ordem arbitrária.

Portanto, o primeiro enfoque foi elaborado em ordem numérica sequencial qualquer das parcelas, da menor para a maior. O segundo foi formado em ordem crescente do número de espécies em cada parcela, ou seja, ordenado da parcela com menor número de espécies para a de maior número de espécies amostradas. O terceiro foi elaborado de maneira inversa ao segundo, ou seja, ordenado da parcela com maior número de espécies para a de menor número. Dessa forma, geraram-se três gráficos distintos, conforme a abordagem para a curva do coletor, para cada intensidade de área amostrada e processo de amostragem, sendo que cada um apresentou todos os tratamentos avaliados.

\section{RESULTADOS E DISCUSSÃO}

No censo florestal dos 25 hectares, foram observados 14.280 indivíduos pertencentes a 113 espécies arbóreas, estas representando 42 famílias botânicas. A família botânica com maior número de indivíduos foi a Lauraceae (3.859 indivíduos), seguida das famílias Aquifoliaceae (1.739 indivíduos), Salinaceae (1.379 indivíduos), Myrtaceae (1.202 indivíduos) e Araucariaceae (1.051 indivíduos).

A espécie com maior número de indivíduos foi Araucaria angustifolia (Bertol.) Kuntze, com 1.051 indivíduos, seguida da espécie Ilex paraguariensis A. St.-Hil. (1.395 indivíduos) e Ocotea odorifera Rohwer (1.309 indivíduos). Essas espécies mais comuns foram amostradas em todos os tratamentos testados na área de estudo. Entretanto, as espécies Citronella paniculata (Mart.) R. A. Howard, uma Cardiopteridaceae (congonha), e Handroanthus albus (Cham.) Mattos, uma Bignoniaceae (ipê-amarelo), não foram amostradas nos tratamentos, exceto no censo florestal. No censo, foi observado apenas 1 indivíduo de cada uma das espécies Citronella paniculata e Handroanthus albus. Nesse sentido, destaca-se o estudo de Longino et al. (2002), que apontaram atenção especial para os efeitos de limiar metodológico empregados em levantamentos com objetivo de avaliação de espécie. Eles sugerem que, quando espécies são ineficientemente amostradas por uma técnica, pode sugerir que são raras ou ausentes.

Observou-se que os tratamentos não representaram todas as espécies existentes na área estudada. Dos tratamentos que tiveram 2 ha de área medida ( $8 \%$ do total), o tratamento que mais se aproximou do número real de espécies ocorrentes na área foi o tratamento T5 $(20 \times 25 \mathrm{~m})$ com processo de distribuição das unidades amostrais de forma aleatória (Tabela 2, 3 e Figura 3), apresentando 80 espécies $(70,8 \%)$. 
Tabela 2. Síntese dos resultados para os tratamentos avaliados em uma área amostrada de dois hectares.

Table 2. Summary of results by sampling treatment evaluated in a sample of two hectares.

\begin{tabular}{|c|c|c|c|c|c|c|c|c|c|}
\hline \multicolumn{2}{|c|}{ Critérios } & $\begin{array}{c}\text { T1 } \\
\text { Censo }\end{array}$ & $\begin{array}{c}\mathrm{T} 2 \\
10 \times 50 \mathrm{~m}\end{array}$ & $\begin{array}{c}\mathrm{T3} \\
10 \times 100 \mathrm{~m}\end{array}$ & $\begin{array}{c}\mathrm{T4} \\
20 \times 20 \mathrm{~m}\end{array}$ & $\begin{array}{c}\text { T5 } \\
20 \times 25 m\end{array}$ & $\begin{array}{c}\text { T6 } \\
20 \times 50 \mathrm{~m}\end{array}$ & $\begin{array}{c}\mathrm{T} 7 \\
30 \times 35 \mathrm{~m}\end{array}$ & $\begin{array}{c}\text { T8 } \\
25 \times 40 m\end{array}$ \\
\hline \multirow{6}{*}{$\begin{array}{l}\frac{0}{\pi} \\
\frac{0}{0} \\
\frac{d}{2}\end{array}$} & Área $\left(m^{2}\right)$ & 250.000 & 500 & 1.000 & 400 & 500 & 1.000 & 1.050 & 1.000 \\
\hline & $\begin{array}{l}\text { Número de indivíduos estimados } \\
(25 \text { ha) }\end{array}$ & 14.280 & 13.838 & 13.475 & 13.663 & 14.013 & 15.100 & 14.313 & 13.338 \\
\hline & Número de espécies & 113 & 75 & 76 & 70 & 80 & 74 & 71 & 68 \\
\hline & Índice de Margalef & 11,71 & 10,56 & 10,74 & 9,86 & 11,25 & 10,29 & 9,94 & 9,61 \\
\hline & Índice $\mathrm{H}^{\prime}$ de Shannon & 3,57 & 3,54 & 3,51 & 3,49 & 3,55 & 3,51 & 3,47 & 3,45 \\
\hline & Índice de Simpson & 0,04 & 0,04 & 0,05 & 0,04 & 0,04 & 0,04 & 0,04 & 0,05 \\
\hline \multirow{6}{*}{ 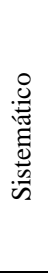 } & Área $\left(\mathrm{m}^{2}\right)$ & 250.000 & 500 & 1.000 & 400 & 500 & 1.000 & 1.050 & 1.000 \\
\hline & $\begin{array}{l}\text { Número de indivíduos estimados } \\
(25 \text { ha) }\end{array}$ & 14.280 & 14.863 & 14.438 & 14.488 & 13.213 & 14.088 & 13.750 & 14.550 \\
\hline & Número de espécies & 113 & 73 & 73 & 71 & 67 & 70 & 73 & 72 \\
\hline & Índice de Margalef & 11,71 & 10,17 & 10,21 & 9,92 & 9,48 & 9,82 & 10,28 & 10,06 \\
\hline & Índice $\mathrm{H}^{\prime}$ de Shannon & 3,57 & 3,52 & 3,49 & 3,47 & 3,48 & 3,5 & 3,53 & 3,44 \\
\hline & Índice de Simpson & 0,04 & 0,04 & 0,04 & 0,05 & 0,04 & 0,04 & 0,04 & 0,05 \\
\hline
\end{tabular}

Tabela 3. Síntese dos resultados para os tratamentos avaliados em uma área amostrada de quatro hectares.

Table 3. Summary of results by sampling treatment evaluated in a sample of four hectares.

\begin{tabular}{|c|c|c|c|c|c|c|c|c|c|}
\hline \multirow{2}{*}{\multicolumn{2}{|c|}{ Critérios }} & T1 & $\mathbf{T 2}$ & T3 & $\mathbf{T 4}$ & T5 & T6 & T7 & T8 \\
\hline & & Censo & $10 \times 50 \mathrm{~m}$ & $10 \times 100 \mathrm{~m}$ & $20 \times 20 m$ & $20 \times 25 m$ & $20 \times 50 \mathrm{~m}$ & 30x35m & $25 \times 40 m$ \\
\hline \multirow{6}{*}{ 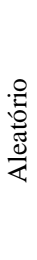 } & Área $\left(m^{2}\right)$ & 250.000 & 500 & 1.000 & 400 & 500 & 1.000 & 1.050 & 1.000 \\
\hline & $\begin{array}{l}\text { Número de indivíduos estimados } \\
(25 \mathrm{ha})\end{array}$ & 14.280 & 14.494 & 13.763 & 14.700 & 13.406 & 13.863 & 14.181 & 14.250 \\
\hline & Número de espécies & 113 & 82 & 87 & 85 & 82 & 90 & 80 & 83 \\
\hline & Índice de Margalef & 11,71 & 10,45 & 11,17 & 10,82 & 10,56 & 11,55 & 10,22 & 10,61 \\
\hline & Índice H' de Shannon & 3,57 & 3,52 & 3,56 & 3,55 & 3,49 & 3,59 & 3,5 & 3,48 \\
\hline & Índice de Simpson & 0,04 & 0,04 & 0,04 & 0,04 & 0,05 & 0,04 & 0,05 & 0,05 \\
\hline \multirow{6}{*}{ 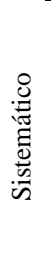 } & Área $\left(\mathrm{m}^{2}\right)$ & 250.000 & 500 & 1.000 & 400 & 500 & 1.000 & 1.050 & 1.000 \\
\hline & $\begin{array}{l}\text { Número de indivíduos estimados } \\
(25 \text { ha) }\end{array}$ & 14.280 & 14.550 & 14.063 & 14.644 & 15.313 & 14.356 & 13.763 & 14.231 \\
\hline & Número de espécies & 113 & 88 & 83 & 87 & 85 & 82 & 85 & 86 \\
\hline & Índice de Margalef & 11,71 & 11,22 & 10,62 & 11,08 & 10,76 & 10,47 & 10,91 & 11 \\
\hline & Índice H' de Shannon & 3,57 & 3,52 & 3,5 & 3,54 & 3,54 & 3,53 & 3,53 & 3,53 \\
\hline & Índice de Simpson & 0,04 & 0,04 & 0,05 & 0,04 & 0,04 & 0,04 & 0,04 & 0,04 \\
\hline
\end{tabular}

A dificuldade em retratar todas as espécies foi apontada por Magurran (2011), que afirma que poucas pesquisas contabilizam todas as espécies, relatando que tempo, custo e especialistas com as habilidades apropriadas de identificação são invariavelmente escassos. Adicionalmente, acrescenta que, em muitos casos, é difícil julgar em que extensão o conjunto de dados é deficiente. Bonetes (2003), ao testar a adequação de vários tamanhos e formas de unidades de amostra variando de $200 \mathrm{~m}^{2}$ a $4.000 \mathrm{~m}^{2}$ para estimar o número de árvores e a área basal em uma Floresta Ombrófila Mista em Chapecó, estado de Santa Catarina, concluiu que, para as espécies agrupadas, as estimativas foram muito precisas para os tamanhos e níveis amostrais testados. No entanto, quando os resultados foram comparados no nível de espécies, nenhum tamanho de parcela produziu estimativas confiáveis, tanto para estimar o estoque como para estudos fitossociológicos.

Analisando a amostragem em 16\% da área total (4 ha), percebe-se que houve um aumento das espécies identificadas pela amostragem. $\mathrm{O}$ tratamento $\mathrm{T} 6$, com processo aleatório e com parcelas de 20 x $50 \mathrm{~m}$, foi o que mais se aproximou do número de espécies observado pelo censo, com 90 espécies identificadas das 113 do censo (79,7\%), ou seja, dobrando a área amostrada, houve um incremento de 8,9\% no número de espécies identificadas. Entretanto, observando apenas o número total de indivíduos estimados pelos tratamentos, sem se ater às espécies, percebe-se que esse tratamento apresentou em média 16,68 indivíduos a menos por hectare em relação ao censo, ou seja, 417 indivíduos a menos na área 
de 25 hectares. De forma geral, observou-se que o processo aleatório para áreas menores amostradas ( 2 ha, $8 \%$ da população) tendeu a apresentar um número maior de espécies na amostragem em relação ao sistemático. Ao passo que, para os 4 ha (16\% da população), o processo sistemático apresentou, na maior parte dos tratamentos, melhor desempenho em relação a essa variável (Figura 3a).

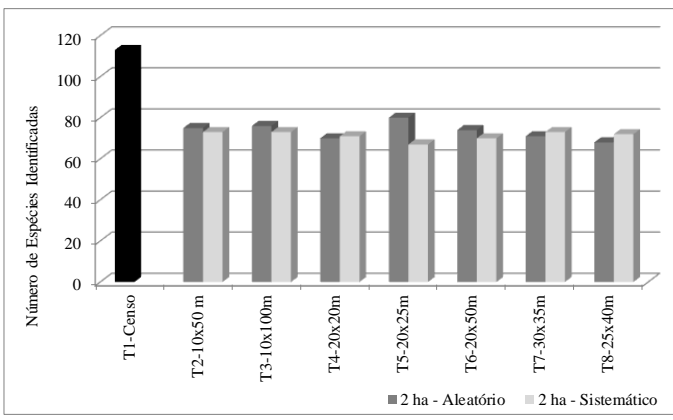

(a) Número de espécies identificadas para 2 hectares;

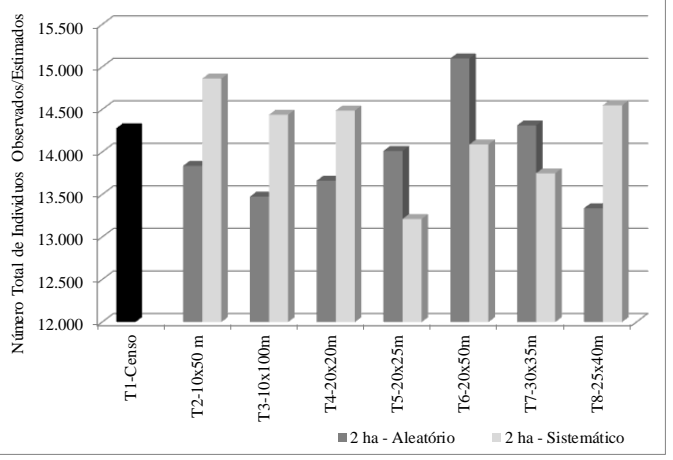

(a) Número total de indivíduos para 2 hectares.

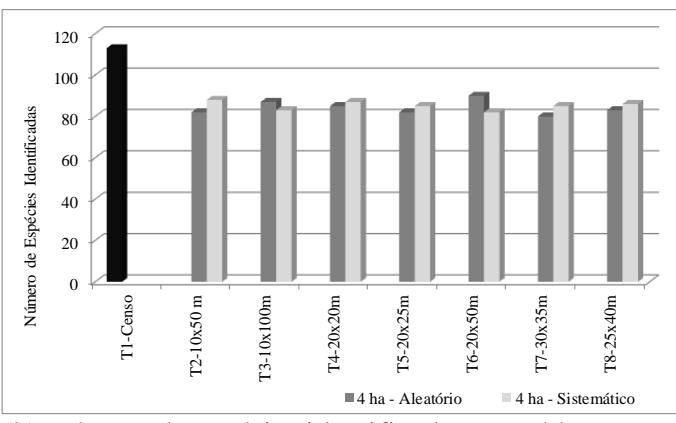

(b) Número de espécies identificadas para 4 hectares;

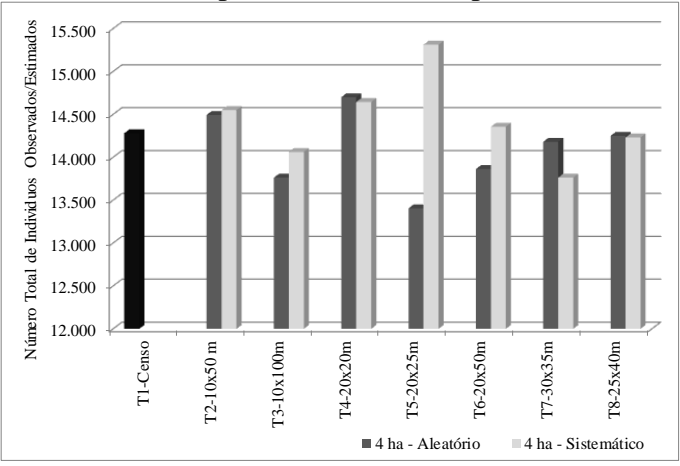

(b) Número total de indivíduos para 4 hectares.

Figura 3. Número total de indivíduos observados e estimados.

Figure 3. Total number of individuals observed and estimated.

Magurran (2011) aponta a existência de dois métodos principais para expressar estimativas de riqueza de espécies - como riqueza de espécies numéricas (número de espécies por um número específico de indivíduos ou biomassa) ou densidade de espécies (número de espécies por unidade de área). Dessa forma, uma vez que a densidade de espécies é um dos principais métodos de avaliação da riqueza das espécies, em trabalhos envolvendo amostragem com essa finalidade, deve ser dada especial atenção a esses resultados. Adicional dificuldade de comparação entre trabalhos foi apontada por Tabarelli e Mantovani (1999), os quais afirmaram que a maioria dos trabalhos não define com clareza o que considera diversidade, de que grupos estão tratando, a escala de abordagem e, principalmente, qual é o controle adotado para as comparações.

Observando apenas em número total de indivíduos estimado, sem se ater às espécies amostradas, o tratamento 8 apresentou apenas 30 indivíduos a mais para os 25 ha (1,2 indivíduos/ha), e o tratamento 7 apresentou 33 indivíduos a menos para os 25 ha (1,32 indivíduos/ha), ambos em relação ao censo (Figura $4 \mathrm{~b}$ ). Já os tratamentos 5 e 6 foram os que apresentaram as maiores superestimativas de números de indivíduos, sendo de 1.033 e 820 nos 25 , ou seja, 41,32 e 32,80 indivíduos/ha, respectivamente, em relação ao censo. Os Índices de Margalef calculados variaram entre 9,48 (tratamento 5 com parcelas de $20 \times 25 \mathrm{~m}$, processo sistemático com amostragem de 2 ha) e 11,51 (tratamento 6 com parcelas de $20 \times 50 \mathrm{~m}$, processo aleatório com amostragem de 4 hectares), tendo sido calculado para o censo o valor de 11,71. O índice de diversidade de Margalef (CLIFFORD; STEPHENSON, 1975) é um índice simples de riqueza de espécies que procura compensar os efeitos de amostragem, dividindo a riqueza, número de espécies registradas, pelo número total de indivíduos da amostra. Magurran (2011) acrescenta que esse índice é fortemente afetado pela intensidade amostral. Prade (2007) afirma que esse índice é considerado o mais antigo e simples para o conceito de diversidade, porém apresenta problemas, visto que nem sempre é possível enumerar todas as espécies de uma comunidade. 
Os Índices de Shannon (H') aplicados nos tratamentos avaliados variaram entre 3,44 (tratamento 8 com parcelas de $25 \times 40 \mathrm{~m}$, processo sistemático com amostragem de 2 ha) e 3,59 (tratamento 6 com parcelas de $20 \times 25 \mathrm{~m}$, processo aleatório com amostragem de 4 ha). O tratamento que mais se aproximou ao valor calculado para o censo $(3,57)$ foi o tratamento 3 , com parcelas de $10 \mathrm{~m} \mathrm{x} 100 \mathrm{~m}$, com processo aleatório e com área amostrada de 4 ha.

Os Índices de Simpson calculados para os tratamentos testados variaram entre 0,04 e 0,05. Os tratamentos aplicados apresentaram um ou outro valor desses citados anteriormente. $\mathrm{O}$ valor do censo foi de 0,04. Essa inflexibilidade para expressar a diversidade de espécies decorre do fato de se levar em consideração a representatividade das espécies com maior valor de importância, sem avaliar a contribuição das demais espécies (MORENO, 2001). Kanieski (2010) complementa que esse índice mostra a probabilidade de que dois indivíduos extraídos ao acaso de uma dada comunidade sejam de espécies diferentes, portanto, é fortemente influenciado pelas espécies mais abundantes da unidade amostral, enquanto é menos sensível à riqueza de espécies (LUDWIG; REYNOLDS, 1988; MAGURRAN, 2011).

Adicionalmente, foi analisada a curva do coletor gerada para todos os tratamentos aplicados. As diferentes abordagens metodológicas testadas neste trabalho possivelmente implicariam diferentes resultados obtidos com a construção da curva para a área em questão. Braun-Blanquet (1932) e MuellerDombois e Ellemberg (1974) citaram que a curva construída pela agregação, ligada ao conceito de área mínima da associação, pressupõe o uso de uma parcela única formada a partir da lista de espécies em uma parcela relativamente pequena, que é gradativamente aumentada (normalmente dobrando seu tamanho) e tem as espécies adicionais contadas. Esse procedimento continua até que a adição de novas áreas não acrescente mais novas espécies ou, ao menos, um número significativo delas.

A figura 4 indica que, para uma mesma amostra, apenas alterando a sequência de entrada na construção do gráfico, os resultados podem gerar levar a diferentes interpretações, confirmando a indicação de Colwell e Coddington (1994) e Martins e Santos (1999) de que a arbitrariedade na ordem de entrada das unidades amostrais na construção da curva poderá produzir resultados distintos.

Percebeu-se que a maior amostragem em termos de área (4 ha) tendeu a trazer resultados mais estáveis para curva do coletor em relação à amostragem de menor área ( 2 ha), resultado este que reforça a afirmação de Scolforo e Mello (2006) de que a estabilização dessa curva é subjetiva, mas que na prática tende a ocorrer à medida que a amostragem se aproxima do censo.

Pode-se observar que, ordenando as parcelas de maneira decrescente considerando o número de espécies, realizou-se uma permuta para que a curva do coletor iniciasse de um número superior de espécies (Figuras 4c, f, i, l), tendendo a atingir mais rapidamente a estabilização no número de espécies. Constatou-se que, para a amostragem de 4 ha, essa tendência ficou mais evidente. Por outro lado, observando o ordenamento das parcelas da que apresentava menor número de espécies para a de maior número de espécies, percebeu-se uma tendência para que a estabilização das espécies não fosse confirmada. Tal fato, possivelmente, sustentou as críticas de vários autores em adotar a curva do coletor como forma de determinar a adequação de uma amostra para estudos de ecologia vegetal (HOPKINS, 1957; RICE; KELTING, 1955; MARTINS; SANTOS, 1999).

Outra questão que ficou explícita foi a dificuldade de identificar e validar de forma visual a estabilização da curva. Para tal constatação, vários autores se dedicaram na busca para definir um ponto de estabilização para a curva do coletor, como, por exemplo, Nappo et al. (1999), Nappo et al. (2000), Gama et al. (2001), Gama et al. (2003), Rondon Neto et al. (2000), Bentes-Gama et al. (2002) e Budke et al. (2004).

Ainda, foi realizada uma avaliação dos tratamentos melhores (Figura 5a-f) e piores (Figura 5g-l) em relação à identificação do número de espécies estarem mais próximo ao valor observado no censo, de forma a considerar a afirmação de Schilling e Batista (2008) de que a ideia de representatividade da curva do coletor está relacionada à indicação de que a composição florística e a densidade de árvores por espécie está adequadamente amostrada.

Para os melhores tratamentos, ou seja, aqueles cujos valores de número de espécies mais se aproximaram aos do censo, a tendência à estabilização foi melhor observada quando da realização do ordenamento das parcelas do maior número de espécies para a parcela de menor número de espécies. Portanto, reforça-se a afirmação de Schilling e Batista (2008) de que qualquer interpretação a respeito da forma da curva, ou de variações nessa forma a partir de um padrão básico esperado, é inválida se essa curva tiver sido gerada a partir de uma ordem arbitrária. Já para o o pior tratamento, de 2 hectares e sistemático com parcelas de $20 \times 25 \mathrm{~m}$, apesar de ter apresentado somente 67 espécies em relação às 113 do censo $(59,29 \%)$, apresentou na curva do coletor a maior tendência a estabilização, conforme verificase na figura 5 i. 

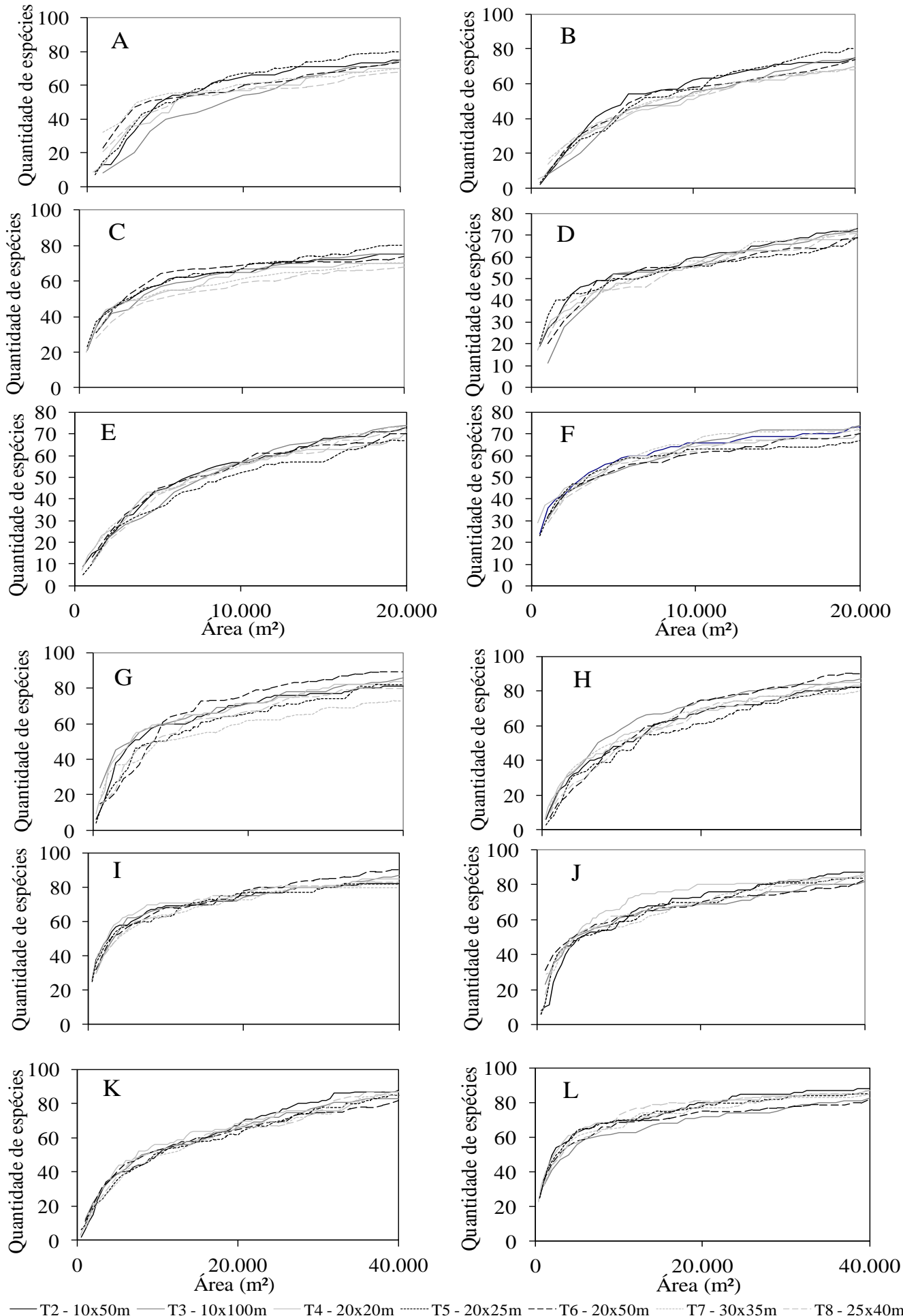

$-\mathrm{T} 2-10 \times 50 \mathrm{~m}-\mathrm{T} 3-10 \times 100 \mathrm{~m}-\mathrm{T} 4-20 \times 20 \mathrm{~m}-\mathrm{T} 5-20 \times 25 \mathrm{~m}-\mathrm{T} 6-20 \times 50 \mathrm{~m}-\mathrm{T} 7-30 \times 35 \mathrm{~m}-\mathrm{T} 8-25 \mathrm{x} 40 \mathrm{n}$ -
Figura 4. Curva do coletor para a área amostrada de 2 hectares (A-F) e de 4 hectares (G-L).

Figure 4. Species accumulation curve to the sampled area of 2 hectares (A-F) and of 4 hectares (G-L).

Sendo: $(\mathrm{a}, \mathrm{g})$ : processo aleatório - ordenado pelo número sequencial; $(\mathrm{b}, \mathrm{h})$ : processo aleatório - ordenado do menor para o maior número de espécies; (c, i): processo aleatório - ordenado do maior para o menor número de espécies; $(\mathrm{d}, \mathrm{j})$ : processo sistemático ordenado pelo número sequencial; $(\mathrm{e}, \mathrm{k})$ : processo sistemático - ordenado do menor para o maior número de espécies; (f, l): processo sistemático - ordenado do maior para o menor número de espécies. 

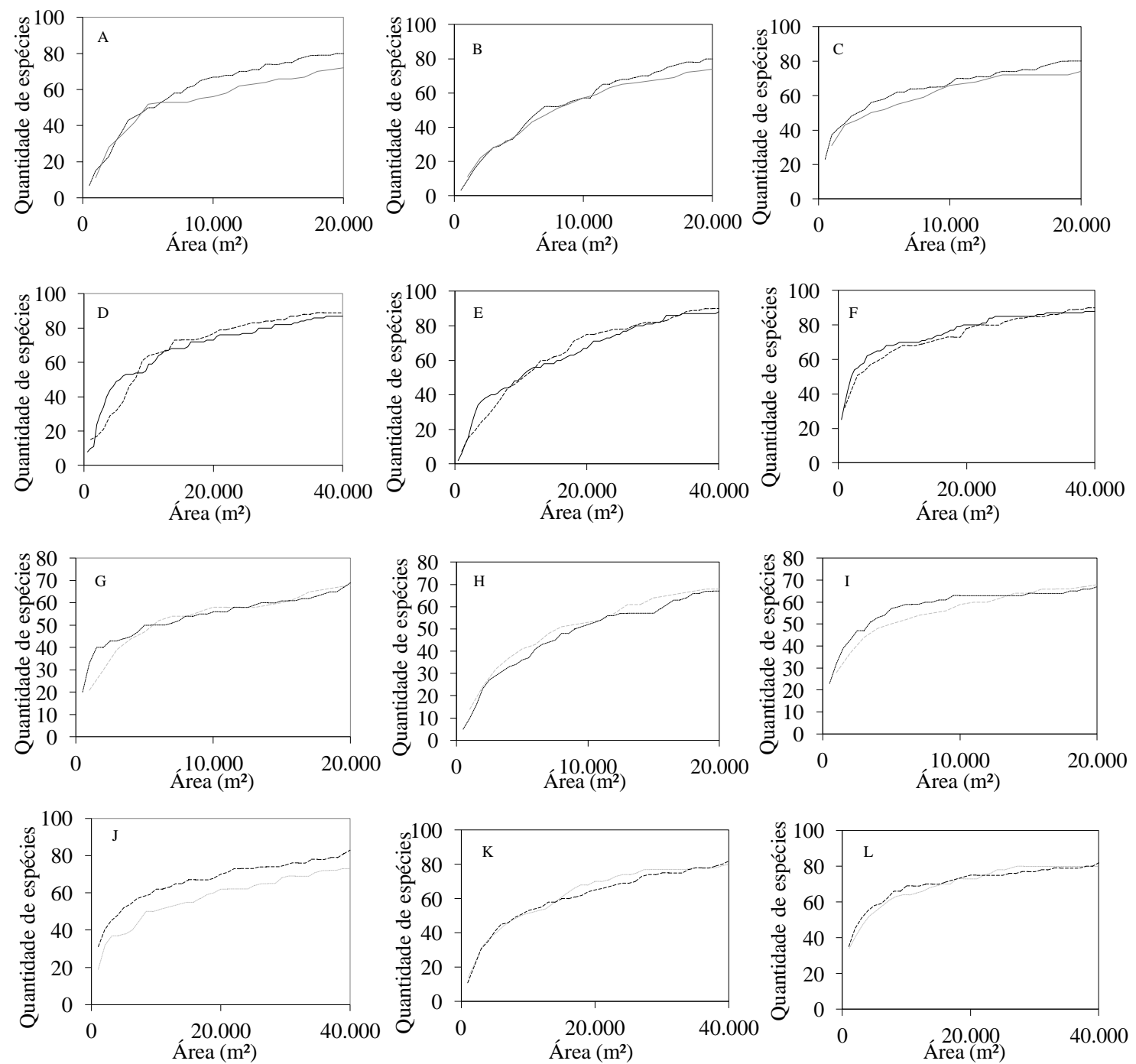

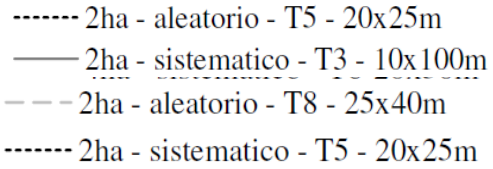

---4 ha - aleatorio - T6 - 20x50m

- 4ha - sistematico - T2 - 10x50m

- 4 ha - aleatorio - T7-30x35m

---4 ha - sistematico - T6-20x50m

Figura 5. Curva do coletor para os melhores tratamentos de 2 hectares e 4 hectares (A-F) e para os piores tratamentos de 2 hectares e 4 hectares (G-L).

Figure 5. Collector curve for better treatments of 2 hectares and 4 hectares and worst treatment of 2 and 4 hectares.

Sendo: (a, g): amostragem em 2 hectares - ordenado pelo número sequencial; (b, h): amostragem em 2 hectares - ordenado do menor para o maior número de espécies; (c, i): amostragem em 2 hectares - ordenado do maior para o menor número de espécies; (d, j): processo sistemático em 4 hectares - ordenado pelo número sequencial; $(\mathrm{e}, \mathrm{k})$ : processo sistemático em 4 hectares - ordenado do menor para o maior número de espécies; (f, 1): processo sistemático em 4 hectares - ordenado do maior para o menor número de espécies.

\section{CONCLUSÕES}

- O processo aleatório tende a representar melhor o número de espécies quando o tamanho da área amostrada foi menor (2 ha), e o sistemático, quando a área amostrada foi maior (4 ha). 
- Os dois melhores tratamentos observados para representação do número de espécies apresentavam 50 metros de comprimento, o primeiro com 20 metros de largura e o segundo com 10 metros de largura, com área amostrada de 4 hectares.

- O índice de Simpson não teve sensibilidade para representar as variações encontradas em relação a todos os tratamentos testados.

- O índice de Shannon apresentou pequena variação para os tratamentos testados, mesmo com cerca de $20 \%$ das espécies não tendo sido identificadas pelo melhor tratamento da amostragem e com $40 \%$ no pior tratamento.

- O índice de Margalef foi o que apresentou a melhor sensibilidade entre os índices testados, para expressar a diversidade de espécies presentes na área em questão.

- Conclui-se ainda que a curva do coletor pode ser influenciada pela ordem de alocação das amostras no gráfico, podendo demonstrar uma situação que não necessariamente reflete a estabilidade na inclusão de novas espécies na amostragem.

\section{REFERÊNCIAS}

BENTES-GAMA, M. M.; SCOLFORO, J. R. S.; GAMA, J. R. V.; OLIVEIRA, A. D. Estrutura e valoração de uma floresta de várzea alta na Amazônia. Cerne, v. 8, p. 88 - 102, 2002.

BONETES, L. Tamanho de parcelas e intensidade amostral para estimar o estoque e índices fitossociológicos em uma Floresta Ombrófila Mista. 111 f. Dissertação (Mestrado em Ciências Florestais) - Universidade Federal do Paraná. Curitiba, 2003.

BRAUN-BLANQUET, J. Plant sociology. New York: McGraw Hill, 1932. 451 p.

BUDKE, J. C.; GIEHL, E. L. H.; ATHAYDE, E. A.; EISINGER, S. M.; ZÁCHIA, R. A. Florística e fitossociologia do componente arbóreo de uma floresta ribeirinha, arroio Passo das Tropas, Santa Maria, RS, Brasil. Acta Botânica Brasílica, São Paulo, v. 18, p. 581 - 589, 2004.

CIELO FILHO, R.; SANTIN, D. A. Estudo florístico e fitossociológico de um fragmento florestal urbano: Bosque dos Alemães, Campinas. Revista Brasileira de Botânica, São Paulo, v. 25, n. 3, p. 291 301, 2002.

CLIFFORD, H. T.; STEPHENSON, W. An Introduction to Numerical Classification. London: Academic Press, 1975. 229 p.

COLWELL, R. K.; CODDINGTON, J. A. Estimating terrestrial biodiversity through extrapolation. Philosophical Transactions: Biological Sciences, Great Britain, v. 345, p. 101 - 118, 1994.

DISPERATI, A. A. Mapeamento florestal da Floresta Nacional de Irati, PR. Curitiba: FUPEF, 1986. $18 \mathrm{p}$.

DURIGAN, G. Estrutura e diversidade de comunidades florestais. In: MARTINS, S. V. Ecologia de florestas tropicais do Brasil, Viçosa, 2009, p. 185 - 215.

ENVIRONMENTAL SYSTEMS RESEARCH INSTITUTE (ESRI). ArcGIS Professional GIS for the desktop, versão 9.3, 2006.

GAMA, J. R. V.; BOTELHO, S. A.; BENTES-GAMA, M. M.; SCOLFORO, J. R. S. Tamanho de parcela e suficiência amostral para estudo da regeneração natural de floresta de várzea na Amazônia. Cerne, Lavras, v. 7, p. 1 - 11, 2001.

GAMA, J. R. V.; BOTELHO, S. A.; BENTES-GAMA, M. M.; SCOLFORO, J. R. S. Estrutura e potencial futuro de utilização da regeneração natural de floresta de várzea alta no município de Afuá, Estado do Pará. Ciência Florestal, Santa Maria, v. 13, p. 71 - 82, 2003.

GUAPYASSÚ, M. S. Caracterização fitossociológica de três fases sucessionais de uma Floresta Ombrófila Densa Submontana, Morretes, Paraná. 165 f. Dissertação (Mestrado em Ciências Florestais) - Universidade Federal do Paraná. Curitiba, 1994.

HOPKINS, B. The concept of minimal area. Journal of Ecology, London, v. 45, p. 441 - 449, 1957. 
KANIESKI, M. R. Caracterização florística, diversidade e correlação ambiental na Floresta Nacional de São Francisco de Paula, Rio Grande do Sul. 99 f. Dissertação (Mestrado em Engenharia Florestal) - Universidade Federal de Santa Maria. Santa Maria, 2010.

LONGINO, J. T.; CODDINGTON, J.; COLWELL, R. K. The ant fauna of a tropical rain forest: estimating species richness three different ways. Ecology, Washington, v. 83, p. 689 - 702, 2002.

LUDWIG, J. A.; REYNOLDS, J. F. Statistical ecology: a primer on methods and computing. New York: John Wiley \& Sons, 1988. 338 p.

MAGURRAN, A. E. Medindo a diversidade biológica. Curitiba: Editora UFPR, 2011. 261 p.

MARTINS, F. R.; SANTOS, F. A. M. Técnicas usuais de estimativa da biodiversidade. Holos, Rio Claro, v. 1 , p. $236-267,1999$.

MAZZA, C. A. S.; MAZZA, M. C. M.; SANTOS, J. E. 2005. SIG aplicado à caracterização ambiental de uma unidade de conservação Floresta Nacional de Irati, Paraná. In: XII SIMPÓSIO BRASILEIRO DE SENSORIAMENTO REMOTO, 12., 2005, Goiânia. Anais... Goiânia, 2005. p. 2251.

MELLO, J. M. Análise comparativa de procedimentos amostrais em um remanescente de floresta nativa no município de Lavras, MG. 99 f. Dissertação (Mestrado em Engenharia Florestal) Universidade Federal de Lavras. Lavras, 1995.

MORAIS FILHO, A. D.; BRAVO, C. V.; ROQUE, R. A. M.; ANDRADE, W. F. Utilização de métodos estatísticos em inventário florestal. Piracicaba: ESALq, 2003.

MOREIRA, C. M. Avaliação de métodos fitossociológicos através de simulações, para um trecho de Cerradão na Estação Ecológica de Assis, SP. 16 f. Dissertação (Mestrado em Recursos Florestais) Escola Superior de Agricultura Luiz de Queiroz, 2007.

MORENO, C. E. Métodos para medir la biodiversidad. Zaragoza, España: M \& T manuales y tesis SEA, 2001. $84 \mathrm{p}$.

MUELLER-DOMBOIS, D.; ELLEMBERG, H. Aims and methods of vegetation analysis. New York: New York Wiley, 1974. 547 p.

NAPPO, M. E.; FONTES, M. A. L.; OLIVEIRA FILHO, A. T. Suficiência amostral e análise do tamanho de parcelas para o estudo da regeneração natural do sub-bosque de povoamentos homogêneos de Mimosa scabrella Benth., em área minerada, em Poços de Caldas, MG. Revista Árvore, Viçosa, v. 23, p. 443 454, 1999.

Regeneração natural em sub-bosque de povoamentos homogêneos de Mimosa scabrella Benth., implantados em áreas mineradas, em Poços de Caldas, MG. Revista Árvore, Viçosa, v. 24, p. 297 - 307 , 2000.

ODA-SOUZA, M.; BATISTA, J. L. F.; RIBEIRO JR., P. J.; RODRIGUES, R. R. Comparação das estruturas de continuidade espacial em quatro formações florestais do Estado de São Paulo. Floresta, Curitiba, n. 3, p. 512 - 522, 2010.

OPTIMBER. FlorExel - Funções Florestais para Microsoft Excel, versão 3.11. OpTimber Otimização e Informática. Curitiba, Paraná, Brasil, 2013. Download (www.optimber.com.br).

PRADE, C. A. Aspectos ecológicos da comunidade fúngica do solo em ambientes ripários com diferentes formas de manejo. 90 f. Tese (Doutorado em Botânica) - Universidade Federal do Rio Grande do Sul. Porto Alegre, 2007.

RICE, E. L.; KELTING, R. W. The species-area curve. Ecology, Washington, v. 36, p. 7 - 11, 1955.

RODE, R. Avaliação florística e estrutural de uma Floresta Ombrófila Mista e de uma vegetação arbórea estabelecida sob um povoamento de Araucaria angustifolia de 60 anos. 159 f. Dissertação (Mestrado em Engenharia Florestal) - Universidade Federal do Paraná. Curitiba, 2008. 
RONDON NETO, R. M.; BOTELHO, S. A.; FONTES, M. A. L.; DAVIDE, A. C.; FARIA, J. M. R. Estrutura e composição florística da comunidade arbustivo-arbórea de uma clareira de origem antrópica em uma floresta estacional semidecídua montana, Lavras, MG. Cerne, Lavras, v. 6, p. 79 - 94, 2000.

SANQUETTA, C. R.; WATZLAWICK, L. F.; CORTE, A. P. D.; FERNANDES, L. A. V.; SIQUEIRA, J. D. P. Inventários Florestais: Planejamento e Execução. Curitiba: Multi-Graphic, 2009. 316 p.

SANTOS, V. K. Uma generalização da distribuição do índice de diversidade generalizada por Good com aplicação em Ciências Agrárias. 57 f. Dissertação (Mestrado em Ciências Agrárias) Universidade Federal de Pernambuco. Pernambuco, 2009.

SAWCZUK, A. R. Florística e estrutura horizontal no período 2002-2008 de um fragmento de floresta ombrófila mista no centro-sul do estado Paraná. 157 f. Dissertação (Mestrado em Engenharia Florestal) - Universidade Estadual do Centro-Oeste, Irati, 2009.

SCHILliNG, A. C.; BATISTA, J. L. F. Curva de acumulação de espécies e suficiência amostral em florestas tropicais. Revista Brasileira de Botância, São Paulo, v. 31, p. 179 - 187, 2008.

SCOLFORO, J. R. S.; MELLO, J. M. de. Inventário Florestal. Lavras: UFLA/Faepe, 2006. 561 p.

SILVA, J. N. M. Eficiência de diversos tamanhos e formas de unidades de amostra aplicadas em inventário florestal na região do baixo Tapajós. 83 f. Dissertação (Mestrado em Ciências Florestais) Universidade Federal do Paraná. Curitiba, 1980.

SINGH, K. D. Reconocimientos forestales: patrones de variación espacial en la selva tropical. Unasylva, Roma, v. 26, p. 18 - 23, 1974.

TABARELLI, M.; MANTOVANI, W. Riqueza de espécies arbóreas na floresta atlântica, SP. Revista Brasileira Botânica, São Paulo, v. 22, p. 217 - 223, 1999.

UBIALLI, J. A.; FIGUEIREDO FILHO, A.; MACHADO, S. A.; ARCE, J. E. Comparação de métodos e processos de amostragem para estudos fitossociológicos em uma floresta ecotonal na região norte matogrossense. Floresta, Curitiba, v. 39, p. 511 - 523, 2009. 
